MATHEMATICS OF COMPUTATION

Volume 68, Number 225, January 1999, Pages 201-216

S 0025-5718(99)01009-1

\title{
IMPROVED ERROR BOUNDS FOR SCATTERED DATA INTERPOLATION BY RADIAL BASIS FUNCTIONS
}

\author{
R. SCHABACK
}

\begin{abstract}
If additional smoothness requirements and boundary conditions are met, the well-known approximation orders of scattered data interpolants by radial functions can roughly be doubled.
\end{abstract}

\section{INTRODUCTION}

Convergence orders of natural cubic spline interpolation to data $f\left(x_{1}\right), \ldots, f\left(x_{N}\right)$ on a mesh

$$
\infty<a=x_{0} \leq x_{1}<\cdots<x_{N} \leq x_{N+1}=b<\infty
$$

are usually provided in three steps [1], [9]. First, in the space

$$
H_{2}^{2}[a, b]=\left\{f:[a, b] \rightarrow \mathbb{R}, f^{\prime \prime} \in L_{2}[a, b]\right\}
$$

where the interpolant minimizes the seminorm

$$
\|f\|_{2}^{2}:=\int_{a}^{b} f^{\prime \prime}(t)^{2} d t
$$

the approximation order is $h^{3 / 2}$ for

$$
h:=\max _{0 \leq j \leq N}\left(x_{j+1}-x_{j}\right),
$$

and this order is optimal on $H_{2}^{2}[a, b]$. In the second step one considers

$$
H_{2}^{4}[a, b]:=\left\{f:[a, b] \rightarrow \mathbb{R}, f^{(4)} \in L_{2}[a, b]\right\}
$$

and imposes the boundary conditions

$$
f^{(j)}(a)=f^{(j)}(b)=0, \quad 2 \leq j \leq 3
$$

to get approximation order $2 \cdot(3 / 2)=3$. Both $f^{(4)} \in L_{2}[a, b]$ and the boundary conditions (1.2) are required to get this order. The third and final step adds the condition $f \in C^{4}[a, b]$ and proves order 4 , which can be shown to be a saturation order, i.e. it cannot be improved by further restrictions on $f$.

Radial basis function techniques can be considered as a generalization of splines to the multivariate case, and here the current status of known approximation orders is comparable to step 1 of the cubic spline case. The available approximation orders are optimal with respect to certain "native" Hilbert spaces generalizing (1.1) in which the interpolant minimizes the norm under all other interpolating functions.

Received by the editor May 10, 1996 and, in revised form, May 21, 1997.

1991 Mathematics Subject Classification. Primary 41A15, 41A25, 41A30, 41A63, 65D10.

(C)1999 American Mathematical Society 
This paper proceeds to Step 2 and thus doubles the approximation order. To do this, the cubic spline case tells us that increased smoothness and certain boundary conditions for $f$ are required. Thus we first have to introduce the "native" Hilbert space and a suitable subspace. These two will replace $H_{2}^{2}[a, b]$ and $H_{2}^{4}[a, b]$ and we shall read off the additional boundary condition from the space of all interpolants (note that (1.2) is the usual property of all natural cubic splines). Since we have to generalize two related spaces and a boundary condition, we have to work out more details than earlier papers. To make the presentation independent of distribution theory and related theories of generalized Fourier transforms, we shall restrict ourselves to Hilbert space techniques and the usual tools of real analysis. We hope that this makes access to native spaces somewhat easier. In this respect, the following three sections provide a new formulation of the basic facts about native spaces.

\section{NATive SPACES}

For natural numbers $d \geq 1$ and $m \geq 0$ let $\mathbb{P}_{m}^{d}$ denote all $d$-variate real polynomials of order up to $m$, and let $\left(\mathbb{P}_{m}^{d}\right)^{\perp}$ be the linear space of all finitely supported linear functionals $\lambda$ of the form

$$
\lambda(f):=\sum_{j=1}^{M} \lambda_{j} f\left(x_{j}\right)
$$

defined on the linear space $C\left(\mathbb{R}^{d}\right)$ that vanish on $\mathbb{P}_{m}^{d}$. Here, $M$ and $x_{1}, \ldots, x_{M} \in \mathbb{R}^{d}$ are allowed to vary freely, but the $x_{j}$ should be distinct.

A continuous function $\Phi: \mathbb{R}^{d} \rightarrow \mathbb{R}$ is conditionally positive definite of order $m$ on $\mathbb{R}^{d}$, if the bilinear form

$$
(\lambda, \mu)_{\Phi}:=\sum_{j=1}^{M} \lambda_{j} \sum_{k=1}^{N} \mu_{k} \Phi\left(x_{j}-y_{k}\right)
$$

is symmetric and positive definite on $\left(\mathbb{P}_{m}^{d}\right)^{\perp}$. Table 1 shows some conditionally positive definite functions with their minimal orders $m$. Any functional $\lambda \in\left(\mathbb{P}_{m}^{d}\right)^{\perp}$ of the form (2.1) generates a continuous function

$$
f_{\lambda}:=\sum_{j=1}^{M} \lambda_{j} \Phi\left(\cdot-x_{j}\right)
$$

on $\mathbb{R}^{d}$. The map $B$

$$
B:\left(\mathbb{P}_{m}^{d}\right)^{\perp} \rightarrow B\left(\left(\mathbb{P}_{m}^{d}\right)^{\perp}\right)=: \mathcal{F}_{0}
$$

defined on $\left(\mathbb{P}_{m}^{d}\right)^{\perp}$ by $B(\lambda)=f_{\lambda}$ is injective, because we have

$$
\mu\left(f_{\lambda}\right)=(\lambda, \mu)_{\Phi}=\lambda\left(f_{\mu}\right)
$$

for all $\lambda, \mu \in\left(\mathbb{P}_{m}^{d}\right)^{\perp}$. Note that $\left(\mathbb{P}_{m}^{d}\right)^{\perp}$ is independent of $\Phi$, while the topology induced by $(\cdot, \cdot)_{\Phi}$ is not. The formula $(2.4)$ shows that $\left(\mathbb{P}_{m}^{d}\right)^{\perp}$ and $\mathcal{F}_{0}:=B\left(\left(\mathbb{P}_{m}^{d}\right)^{\perp}\right)$ form a dual pair, and this one-to-one correspondence between functionals and functions is a basic feature of spaces behind radial basis functions. Furthermore, we remark that the functions $f_{\lambda}$ from $\mathcal{F}_{0}$ are the interpolants that occur in all applications. Thus the space $\mathcal{F}_{0}$ arises very naturally, and we have to investigate the spaces of functions that can be approximated by functions from $\mathcal{F}_{0}$. This in turn requires knowledge of completions of $\mathcal{F}_{0}$ with respect to various topologies. 
TABLE 1. Radial basis functions and Fourier transforms

\begin{tabular}{l||l} 
radial basis function $\Phi(x)$ & $\hat{\Phi}(\omega)$ in $\mathbb{R}^{d}$ \\
\hline \hline$(-1)^{\lceil\nu / 2\rceil}\|x\|^{\nu}, \nu>0, \nu \notin 2 \mathbb{N} m \geq\lceil\nu / 2\rceil$ & $2^{d+\nu} \pi^{d / 2} \frac{\Gamma((d+\nu) / 2)}{\Gamma(-\nu / 2)}\|\omega\|^{-d-\nu}$ \\
\hline$(-1)^{k+1}\|x\|^{2 k} \log \|x\|, k \in 2 \mathbb{N} m>k$ & $2^{d+2 k-1} \pi^{d / 2} \Gamma(d / 2+k) k !\|\omega\|^{-d-2 k}$ \\
\hline$e^{-\alpha\|x\|^{2}}, \alpha>0, m \geq 0$ & $\left(\frac{\pi}{\alpha}\right)^{d / 2} e^{-\|\omega\|^{2} /(4 \alpha)}$ \\
\hline$(-1)^{\lceil\nu / 2\rceil}\left(c^{2}+\|x\|^{2}\right)^{\nu / 2}$, & $(-1)^{\lceil\nu / 2\rceil} \frac{2 \pi^{d / 2}}{\Gamma(-\nu / 2)} K_{(d+\nu) / 2}(c\|\omega\|)$ \\
$\nu>0, \nu \notin 2 \mathbb{N}, c \neq 0, m \geq\lceil\nu / 2\rceil$ & $\times\left(\frac{\|\omega\|}{2 c}\right)^{-(d+\nu) / 2}$ \\
\hline $\begin{array}{l}\left(c^{2}+\|x\|^{2}\right)^{\nu / 2}, m \geq 0 \\
-d<\nu<0, \nu \notin 2 \mathbb{Z}, c \neq 0\end{array}$ & $\frac{2 \pi^{d / 2}}{\Gamma(-\nu / 2)} K_{(d+\nu) / 2}(c\|\omega\|)\left(\frac{\|\omega\|}{2 c}\right)^{-(d+\nu) / 2}$
\end{tabular}

However, there is a specific topology that comes for free from the definition of $\mathcal{F}_{0}$, namely the topology induced by

$$
\left(f_{\lambda}, f_{\mu}\right)_{\Phi}=(\lambda, \mu)_{\Phi}
$$

for all $\lambda, \mu \in\left(\mathbb{P}_{m}^{d}\right)^{\perp}$. The completion $\mathcal{F}$ of $\mathcal{F}_{0}$ with respect to this topology (that is induced by $\Phi$ itself) will be called the native space for interpolation by translates of $\Phi$. The next sections are devoted to the study of $\mathcal{F}$ by Hilbert space and Fourier transform techniques, in order to provide the fundamentals for improved error bounds. At this stage, however, we can already read off (2.3), (2.4), and (2.5) what will later lead to the proper boundary condition in native spaces.

Lemma 2.1. Two functions $f_{\lambda}$ and $f_{\mu}$ are orthogonal, if $f_{\lambda}$ coincides with a polynomial from $\mathbb{P}_{m}^{d}$ on the support of $\mu$ or $f_{\mu}$ coincides with a polynomial from $\mathbb{P}_{m}^{d}$ on the support of $\lambda$.

Before we proceed any further in the investigation of native spaces, we have to describe the interpolation process and the form of its error bounds.

Interpolation of data $f_{1}, \ldots, f_{M}$ on a set $X=\left\{x_{1}, \ldots, x_{M}\right\} \subset \mathbb{R}^{d}$ of $M$ distinct points requires $X$ to be $\mathbb{P}_{m}^{d}$-nondegenerate, i.e. a polynomial from $\mathbb{P}_{m}^{d}$ which vanishes on $X$ must be identically zero. Then one looks for an interpolant

$$
s_{X, f}=p+f_{\lambda}=\sum_{j=1}^{Q} \beta_{j} p_{j}+\sum_{k=1}^{M} \lambda_{k} \Phi\left(\cdot-x_{k}\right)
$$


with $p \in \mathbb{P}_{m}^{d}$, a basis $p_{1}, \ldots, p_{Q}$ of $\mathbb{P}_{m}^{d}$, and $\lambda \in\left(\mathbb{P}_{m}^{d}\right)^{\perp}$ supported on $X$. The interpolation conditions

$$
s_{X, f}\left(x_{j}\right)=p\left(x_{j}\right)+f_{\lambda}\left(x_{j}\right)=f_{j}, \quad 1 \leq j \leq M
$$

can be written in obvious matrix form as

$$
\left(\begin{array}{cc}
A & P \\
P^{T} & 0
\end{array}\right)\left(\begin{array}{c}
\lambda \\
\beta
\end{array}\right)=\left(\begin{array}{l}
f \\
0
\end{array}\right)
$$

with $A=\left(\Phi\left(x_{j}-x_{k}\right)\right)_{1 \leq j, k \leq M}, P=\left(p_{k}\left(x_{j}\right)\right)_{\substack{1 \leq j \leq M \\ 1 \leq k \leq Q}}$. The system (2.7) is uniquely solvable, because $\Phi$ is conditionally positive definite of order $m$ and $X$ is $\mathbb{P}_{m}^{d}$ nondegenerate. Thus there exists a Lagrange basis $u_{1}, \ldots, u_{M}$ for the space of functions (2.6) related to interpolation on $X$, such that

$$
s_{X, f}=\sum_{j=1}^{M} u_{j} f_{j}
$$

and the functions $u_{j}$ solve the system

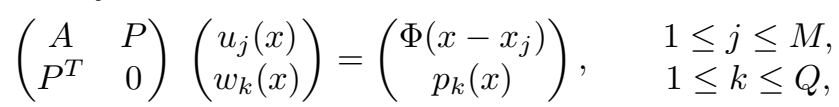

for all $x \in \mathbb{R}^{d}$. The interpolation error now takes a very simple form:

$$
f(x)-s_{X, f}(x)=f(x)-\sum_{j=1}^{M} u_{j}(x) f\left(x_{j}\right)=: \delta_{(x)} f
$$

with a functional $\delta_{(x)} \in\left(\mathbb{P}_{m}^{d}\right)^{\perp}$. This leads to the error bound

$$
\left|f(x)-s_{X, f}(x)\right| \leq\left\|\delta_{(x)}\right\|_{\Phi}\|f\|_{\Phi}
$$

for $x \in \mathbb{R}^{d}$ and $f \in F$, splitting the effect of $x$ and $f$. The nonnegative function $P(x)=P_{X}(x)$ defined by

$$
\begin{aligned}
P_{X}^{2}(x) & =\left\|\delta_{(x)}\right\|_{\Phi}^{2}=\left(\delta_{(x)}, \delta_{(x)}\right)_{\Phi} \\
& =\Phi(0)-2 \sum_{j=1}^{M} u_{j}(x) \Phi\left(x-x_{j}\right) \\
& +\sum_{j, k=1}^{M} u_{j}(x) u_{k}(x) \Phi\left(x_{j}-x_{k}\right)
\end{aligned}
$$

is the power function associated to interpolation on $X$ by $\Phi$-translates, and the error bound (2.9) now reads as

$$
\left|f(x)-s_{X, f}(x)\right| \leq\|f\|_{\Phi} P_{X}(x)
$$

where $s$ and $P$ depend on $X$ and $\Phi$. This is the usual first-stage error bound in the sense of the introduction. We note in passing that the function

$$
\Psi(x, y):=\left(\delta_{(x)}, \delta_{(y)}\right)_{\Phi}
$$

is (in a slightly generalized sense) unconditionally positive definite on $\Omega \backslash X$.

By rather complicated techniques (see [4], [5], [6], [8]) one can bound $P^{2}(x)$ by functions $F(h)=F_{\Phi}(h)$ of the density

$$
h=\sup _{y \in \Omega} \min _{x_{j} \in X}\left\|y-x_{j}\right\|
$$


TABLE 2. All entries are modulo factors that are independent of $r$ and $h$, but possibly dependent on parameters of $\Phi$

\begin{tabular}{|c|c|}
\hline$\Phi(x)=\phi(r), r=\|x\|_{2}$ & $F(h)$ \\
\hline $\begin{array}{l}r^{\beta}, \beta \in \mathbb{R}_{>0} \backslash 2 \mathbb{N} \\
\text { thin-plate splines }\end{array}$ & $\begin{array}{c}h^{\beta} \\
{[10]}\end{array}$ \\
\hline $\begin{array}{c}(-1)^{1+\beta / 2} r^{\beta} \log r, \quad \beta \in 2 \mathbb{N} \\
\text { thin-plate splines }\end{array}$ & $\begin{array}{l}h^{\beta} \\
{[10]}\end{array}$ \\
\hline$\left(\gamma^{2}+r^{2}\right)^{\beta / 2}, \beta \in \mathbb{R} \backslash 2 \mathbb{N}_{\geq 0}$ & $e^{-\frac{\delta}{h}}$ \\
\hline Multiquadrics & $\delta>0$ \\
\hline $\begin{array}{c}e^{-\beta r^{2}}, \beta>0 \\
\text { Gaussians }\end{array}$ & $\begin{array}{c}e^{-\frac{\delta}{h^{2}}} \\
\delta>0[6]\end{array}$ \\
\hline $\begin{array}{c}\frac{2 \pi^{d / 2}}{\Gamma(k)} K_{k-d / 2}(r)(r / 2)^{k-d / 2} \\
2 k>d \\
\text { Sobolev splines }\end{array}$ & $\begin{array}{c}h^{2 k-d} \\
\text { as in }[10]\end{array}$ \\
\hline
\end{tabular}

of $X$ in a compact domain $\Omega$, but we do not want to elaborate these facts (see Table 2 and [7] for full details). The final goal of our paper is to introduce a stronger norm $\|\cdot\|_{\Phi * \Phi}$ on a subspace $\mathcal{H}$ of the completion $\mathcal{F}$ of $F$, and to prove that

$$
\left|f(x)-s_{X, f}(x)\right| \leq\|f\|_{\Phi * \Phi} P(x)\|P\|_{L_{2}(\Omega)}
$$

for a compact domain $\Omega \subset \mathbb{R}^{d}$ and all $f \in \mathcal{H}$. This will roughly double the approximation orders, and it corresponds to Step 2 in the sense of Section 1.

The proper definition of $\mathcal{H}$ must include certain "boundary conditions", and the connection to $L_{2}(\Omega)$ is by no means evident at this stage. These things require a somewhat detailed analysis of native spaces and boundary conditions when everything is restricted to a bounded domain $\Omega$.

\section{LOCALIZATION, EXTENSION AND BOUNDARY CONDITIONS}

To be able to study boundary conditions, we introduce a subset $\Omega \subseteq \mathbb{R}^{d}$ which is supposed to contain the centers $x_{1}, \ldots, x_{M}$ of functionals of the form (2.1). We only assume that $\Omega$ is $\mathbb{P}_{m}^{d}$-nondegenerate, i.e. there is no nontrivial polynomial in $\mathbb{P}_{m}^{d}$ that vanishes on $\Omega$. Thus $\Omega$ may, for instance, be finite, countable (with or without finite accumulation points), a bounded open set or $\mathbb{R}^{d}$ itself, and no further restrictions are made at this point. Then $\left(\mathbb{P}_{m}^{d}\right)_{\Omega}^{\perp}$ is the subspace of $\left(\mathbb{P}_{m}^{d}\right)^{\perp}$ with functionals having support in $\Omega$, and the functions $f_{\lambda}=B(\lambda)$ from the space

$$
\mathcal{F}_{0, \Omega}=B\left(\left(\mathbb{P}_{m}^{d}\right)_{\Omega}^{\perp}\right)
$$

are the approximants whose limits we have to study. The completion of $\mathcal{F}_{0, \Omega}$ with respect to $(\cdot, \cdot)_{\Phi}$ will then form the native space $\mathcal{F}_{\Omega}$. 
Elements of Hilbert space completions are usually defined as equivalence classes of Cauchy sequences, and thus there is no apparent interpretation of elements $f$ of the native space $\mathcal{F}_{\Omega}$ as functions. At the same time, there is no direct meaning of elements of

$$
\mathcal{L}_{\Omega}=\operatorname{clos}_{(\cdot, \cdot)_{\Phi}}\left(\mathbb{P}_{m}^{d}\right)_{\Omega}^{\perp}
$$

as functionals supported on $\Omega$. But the identity (2.4) will carry over to the completions by continuity, and this makes $\mathcal{L}_{\Omega}$ and $\mathcal{F}_{\Omega}$ a dual pair in the sense that

$$
\lambda(f):=\left(\lambda, B^{-1}(f)\right)_{\Phi}=(B(\lambda), f)_{\Phi}
$$

is meaningful for all $\lambda \in \mathcal{L}_{\Omega}, f \in \mathcal{F}_{\Omega}$ and the continuous extension of the isometry $B$ to the respective completions.

Theorem 3.1. Each element $f$ from the native space $\mathcal{F}_{\Omega}$ has an interpretation as a function on $\Omega$, and this interpretation is unique modulo polynomials in $\mathbb{R}_{m}^{d}$.

Proof. We fix a set $\Xi=\left\{\xi_{1}, \ldots, \xi_{Q}\right\} \subseteq \Omega$ with $Q=\operatorname{dim} \mathbb{P}_{m}^{d}=\left(\begin{array}{c}m-1-d \\ d\end{array}\right)$ that is $\mathbb{P}_{m}^{d}$-unisolvent, i.e. there is a Lagrange basis $p_{1}, \ldots, p_{Q}$ of $\mathbb{P}_{m}^{d}$ that satisfies

$$
p(\cdot)=\sum_{j=1}^{Q} p_{j}(\cdot) p\left(\xi_{j}\right)
$$

for all $p \in \mathbb{P}_{m}^{d}$. Then for each $x \in \Omega$ the functional

$$
\delta_{(x)}: f \Rightarrow f(x)-\sum_{j=1}^{Q} p_{j}(x) f\left(\xi_{j}\right)
$$

is in $\left(\mathbb{P}_{m}^{d}\right)_{\Omega}^{\perp}$ and specializes $(2.8)$ to $X=\Xi$. Then one can define

$$
f(x):=\left(B^{-1}(f), \delta_{(x)}\right)_{\Phi}
$$

for all $f \in \mathcal{F}_{\Omega}$ and $x \in \Omega$, assigning function values to the abstract element $f$. It is easy to verify that this definition is consistent with (3.1) in the sense that the usual application of a functional $\mu$ also yields

$$
\mu(f)=\left(B^{-1}(f), \mu\right)_{\Phi}
$$

for all $\mu \in\left(\mathbb{P}_{m}^{d}\right)_{\Omega}^{\perp}$. Any other assignment of function values to $f$ must satisfy this identity, and thus the difference of two assignments is a function $g$ with $\mu(g)=0$ for all $\mu \in\left(\mathbb{P}_{m}^{d}\right)^{\perp}$. Setting $\mu=\delta_{(x)}$ here, we get that $g$ is in $\mathbb{P}_{m}^{d}$, proving the theorem.

Note that (3.3) is a special assignment of function values such that $f(\Xi)=\{0\}$, because $\delta_{\left(\xi_{j}\right)}=0$ for $j=1, \ldots, Q$. Any other assignment can be generated in practice by additional polynomial interpolation on $\Xi$.

Since $\left(\mathbb{P}_{m}^{d}\right)_{\Omega}^{\perp}$ is a subspace of $\left(\mathbb{P}_{m}^{d}\right)_{\mathbb{R}^{d}}$ the completions $\mathcal{L}_{\Omega}$ and $\mathcal{L}_{\mathbb{R}^{d}}$ satisfy $\mathcal{L}_{\Omega} \subseteq$ $\mathcal{L}_{\mathbb{R}^{d}}$ in the sense that there is an injection $J: \mathcal{L}_{\Omega} \rightarrow \mathcal{L}_{\mathbb{R}^{d}}$. Then for all $f \in \mathcal{F}_{\Omega}$ the element $J B^{-1} f$ is in $\mathcal{L}_{\mathbb{R}^{d}}$ and $B J B^{-1} f$ is in $\mathcal{F}_{\mathbb{R}^{d}}$. Because

$$
\lambda\left(B J B^{-1}\right)=\left(\lambda, J B^{-1} f\right)_{\Phi}=\left(\lambda, B^{-1} f\right)_{\Phi}=\lambda(f)
$$


for all $\lambda \in\left(\mathbb{P}_{m}^{d}\right)_{\Omega}^{\perp}$, we see that assignments of function values to $f$ and $B J B^{-1} f$ must coincide on $\Omega$ up to a polynomial in $P_{m}^{d}$. If we denote the interpolating polynomial to $f$ on $\Xi$ by $p_{f}$, then we can define

$$
f_{e}:=p_{f}+B J B^{-1} f
$$

and see that

$$
\left.f_{e}\right|_{\Omega}=\left.f\right|_{\Omega}
$$

This proves an extension theorem first observed by Iske [2], [3].

Theorem 3.2. Any element $f$ of a native space $\mathcal{F}_{\Omega}$ has a canonical extension $f_{e}$ to a function on $\mathbb{R}^{d}$ which lies in $\mathcal{F}_{\mathbb{R}^{d}}$. This furnishes an isometric imbedding of $\mathcal{F}_{\Omega}$ into $\mathcal{F}_{\mathbb{R}^{d}}$.

This extension theorem implicitly contains boundary conditions for functions $f \in \mathcal{F}_{\Omega}$. In case of cubic splines and $\Omega=[a, b]$ it turns out that $\mathcal{F}_{\Omega}$ coincides with $H_{2}^{2}[a, b] / \mathbb{P}_{2}^{1}$ with inner product $\left(f^{\prime \prime}, g^{\prime \prime}\right)_{L_{2}[a, b]}$, and the canonical extensions of $\mathbb{P}_{2}^{1}$-equivalence classes of functions from $\mathcal{F}_{\Omega}$ are linear in $(-\infty, a]$ and $[b, \infty)$. For functions $f$ with additional smoothness (e.g. $f \in H_{2}^{4}[a, b]$ ) this implies the boundary conditions $f^{(j)}(a)=f^{(j)}(b)=0, j=2,3$.

However, the boundary conditions are by no means apparent in general. Thus we now express them by orthogonality relations that will be useful for other purposes, too.

If $\mathcal{F}_{\Omega}^{0}:=B J B^{-1} \mathcal{F}_{\Omega}$ is the embedded image of $\mathcal{F}_{\Omega}$ in $\mathcal{F}_{\mathbb{R}^{d}}$, its orthogonal complement is given by the following result.

Theorem 3.3. In $\mathcal{F}_{\mathbb{R}^{d}}$, the spaces $\mathcal{F}_{\Omega}^{0}$ and

$$
\mathcal{F}_{\Omega}^{\perp}:=\left\{g \in \mathcal{F}_{\mathbb{R}^{d}}:(J \lambda)(g)=0 \text { for all } \lambda \in \mathcal{L}_{\Omega}\right\}
$$

yield an orthogonal decomposition.

Proof. It is straightforward to prove orthogonality. To find that $\mathcal{F}_{\Omega}^{\perp}$ is indeed the full orthogonal complement of $\mathcal{F}_{\Omega}^{0}$, let $g \in \mathcal{F}_{\mathbb{R}^{d}}$ be orthogonal to $\mathcal{F}_{\Omega}^{0}$. Then

$$
(g, B J \lambda)_{\Phi}=(J \lambda)(g)=0 \text { for all } \lambda \in \mathcal{L}_{\Omega}
$$

implies $g \in \mathcal{F}_{\Omega}^{\perp}$.

There are two other formulations that may be somewhat more handy.

Corollary 3.4. The orthogonal complement of a native space $\mathcal{F}_{\Omega}$, when embedded in $\mathcal{F}_{\mathbb{R}^{d}}$, consists of all functions $g$ on $\mathbb{R}^{d}$ that are assignments of function values to some element of $\mathcal{F}_{\mathbb{R}^{d}}$ such that $g$ coincides with a polynomial from $\mathbb{P}_{m}^{d}$ on $\Omega$.

Corollary 3.5. A function $f_{\lambda}$ of the form (2.3) is orthogonal to an element $g \in \mathcal{F}_{\Omega}$ if $g$ takes values of a polynomial from $\mathbb{P}_{m}^{d}$ on the support of $\lambda$.

This generalizes Lemma 2.1 and can possibly be used for multilevel methods with orthogonality between levels. Another question related to orthogonality concerns the support of generalized functionals $\lambda \in \mathcal{L}_{\Omega}$. Of course, for any $\lambda \in\left(\mathbb{P}_{m}^{d}\right)_{\Omega}^{\perp}$ we have

$$
\lambda(u)=\left(f_{\lambda}, u\right)_{\Phi}=0
$$

for all $u \in \mathcal{F}_{\mathbb{R}^{d}}$ that vanish or coincide with a $\mathbb{P}_{m}^{d}$-polynomial on $\Omega$. This property extends to $\mathcal{L}_{\Omega}$ by continuity and is a generalization of the statement " $\operatorname{supp}(\lambda) \subseteq \Omega$ ". 
So far we have defined the native space $\mathcal{F}_{\Omega}$ as the completion of $\left(\mathbb{P}_{m}^{d}\right)_{\Omega}^{\perp}$ with respect to $(\cdot, \cdot)_{\Phi}$. We now turn to another equivalent space introduced by Madych and Nelson.

Theorem 3.6. The space $\mathcal{F}_{\Omega} \oplus \mathbb{P}_{m}^{d}$ is isometrically isomorphic to the space $\mathcal{G}_{\Omega}$ of all real-valued functions $f$ on $\Omega$ for which there is a constant $c_{f} \geq 0$ such that

$$
|\lambda(f)| \leq c_{f}\|\lambda\|_{\Phi}
$$

for all $\lambda \in\left(\mathbb{P}_{m}^{d}\right)_{\Omega}^{\perp}$. The topology on $\mathcal{G}_{\Omega}$ is defined by the seminorm

$$
|f|_{\Phi}:=\sup _{\substack{\lambda \in\left(\mathbb{P}^{d}\right), \frac{1}{\Omega} \\ \lambda \neq 0}} \frac{|\lambda(f)|}{\|\lambda\|_{\Phi}} \leq C_{f},
$$

which yields a norm on $\mathcal{G}_{\Omega} / \mathbb{P}_{m}^{d}$ that agrees with the norm $\|\cdot\|_{\Phi}$ on $\mathcal{F}_{\Omega} \cong \mathcal{G}_{\Omega} / \mathbb{P}_{m}^{d}$.

Proof. The statements concerning the seminorm on $\mathcal{G}_{\Omega}$ and the norm on $\mathcal{G}_{\Omega} / \mathbb{P}_{m}^{d}$ are easy to prove. For every assignment of function values to $f \in \mathcal{F}_{\Omega}$ we have

$$
|\lambda(f)| \leq\|f\|_{\Phi}\|\lambda\|_{\Phi}
$$

for all $\lambda \in \mathcal{L}_{\Omega}$, and for the fixed assignment of function values in the sense of (3.3) we get $\mathcal{F}_{\Omega} \subseteq \mathcal{G}_{\Omega}$. Furthermore, from $\mathbb{P}_{m}^{d} \subseteq \mathcal{G}_{\Omega}$ and $\mathbb{P}_{m}^{d} \cap \mathcal{F}_{\Omega}=\emptyset$ and $\|f\|_{\Phi}=|f|_{\Phi}$ for $f \in \mathcal{F}_{\Omega}$ satisfying (3.3) we see that

$$
\mathcal{F}_{\Omega} \oplus \mathbb{P}_{m}^{d} \subset \mathcal{G}_{\Omega}, \quad \mathcal{F}_{\Omega} \subset \mathcal{G}_{\Omega} / \mathbb{P}_{m}^{d} .
$$

The definition of $\mathcal{G}_{\Omega}$ implies that $\mathcal{G}_{\Omega}$ and $\mathcal{G}_{\Omega} / \mathbb{P}_{m}^{d}$ are closed. To prove that $\mathcal{F}_{\Omega}$ is not a proper subspace of $\mathcal{G}_{\Omega} / \mathbb{P}_{m}^{d}$, we consider an element $g+\mathbb{P}_{m}^{d}$ and the functional

$$
\lambda \mapsto \lambda(g)
$$

on $\left(\mathbb{P}_{m}^{d}\right)_{\Omega}^{\perp}$. This is continuous and extends to $\mathcal{L}_{\Omega}$. By the Fischer-Riesz theorem on $\mathcal{L}_{\Omega}$ there is an element $\mu_{g} \in \mathcal{L}_{\Omega}$ such that

$$
\lambda(g)=\left(\lambda, \mu_{g}\right)_{\Phi}=\lambda\left(B\left(\mu_{g}\right)\right)
$$

for all $\lambda \in \mathcal{L}_{\Omega}$. This implies that $g$ and $B\left(\mu_{g}\right) \in \mathcal{F}_{\Omega}$ coincide on $\Omega$ up to a polynomial in $\mathbb{P}_{m}^{d}$, and thus $g+\mathbb{P}_{m}^{d}=B\left(\mu_{g}\right)+\mathbb{P}_{m}^{d}$ as equivalence classes of functions on $\Omega$. This proves the assertion.

\section{Fourier TRANSFORMS}

To get more information out of the results of the preceding section we now add an assumption that looks very restrictive but is satisfied in all practical cases. For any $\lambda, \mu \in\left(\mathbb{P}_{m}^{d}\right)^{\perp}$ we assume that there is a representation of

$$
\begin{aligned}
(\lambda, \mu)_{\Phi} & =\sum_{j=1}^{M} \sum_{k=1}^{N} \lambda_{j} \mu_{k} \Phi\left(x_{j}-y_{k}\right) \\
& =(2 \pi)^{-d} \int_{\mathbb{R}^{d}} \hat{\Phi}(\omega)\left(\sum_{j=1}^{M} \lambda_{j} e^{i x_{j}^{T} \omega}\right)\left(\sum_{k=1}^{N} \bar{\mu}_{k} e^{-i x_{k}^{T} \omega}\right) d \omega
\end{aligned}
$$

as a Lebesgue integral with a nonnegative function

$$
\hat{\Phi}: \mathbb{R}^{d} \backslash\{0\} \rightarrow \mathbb{R}_{\geq 0}
$$


that vanishes at most on a set of measure zero. For positive orders $m$ of conditional positive definiteness of $\Phi$, the function $\hat{\Phi}$ may have a singularity at zero. This is cancelled by the zeros of order $m$ at zero of the functions

$$
\hat{\lambda}(\omega):=\sum_{j=1}^{M} \lambda_{j} e^{i x_{j}^{T} \omega},
$$

as results from the property $\lambda \in\left(\mathbb{P}_{m}^{d}\right)^{\perp}$ via Taylor expansion of the exponential. More precisely, we assume $\hat{\Phi}$ to have a singularity

$$
\hat{\Phi}(\omega) \approx\|\omega\|^{-d-\beta_{0}}
$$

for $\omega$ near zero, and we assume $m$ to be minimal or $\beta_{0}$ to be maximal under the restriction

$$
\beta_{0} \leq 2 m
$$

that makes the integral well-defined near zero. Table 1 shows the functions $\hat{\Phi}$ for various choices of $\Phi$.

As a referee correctly pointed out, the assumption (4.1) contains a subtlety, because it insists on $\hat{\Phi}$ being a classical function, thus excluding $\hat{\Phi}$ from being a fully general $L_{2}\left(\mathbb{R}^{d}\right)$ function or a distribution. Furthermore, the existence of the integral implicitly assumes some hidden decay condition on $\hat{\Phi}$ that is related to the order $m$ of conditional positive definiteness. We could elaborate on these delicate points, but we want to avoid detours.

We have adopted generalized Fourier transform notation in (4.1) and (4.3), but we do not require any knowledge of generalized Fourier transform theory in what follows. All the integrals that arise will exist classically. Rewriting (4.1) with (4.3) in the form

$$
(\lambda, \mu)_{\Phi}=(2 \pi)^{-d} \int_{\mathbb{R}^{d}} \hat{\Phi}(\omega) \hat{\lambda}(\omega) \overline{\hat{\mu}(\omega)} d \omega
$$

we shall assume that

$$
\hat{\lambda} \sqrt{\hat{\Phi}} \in L_{2}\left(\mathbb{R}^{d}\right) \text { for all } \lambda \in\left(\mathbb{P}_{m}^{d}\right)^{\perp} .
$$

This implies existence of the integral in (4.1).

We now have a tool to connect native spaces with $L_{2}$ spaces. In particular,

$$
C: \lambda \Rightarrow \hat{\lambda} \sqrt{\hat{\Phi}}
$$

maps $\left(\mathbb{P}_{m}^{d}\right)^{\perp}$ isometrically into $L_{2}\left(\mathbb{R}^{d}\right)$, if we define the $L_{2}$ inner product as usual, but with the factor $(2 \pi)^{-d}$.

Theorem 4.1. Assume that (4.1)-(4.6) hold. Then the map $C$ extends by continuity to $\mathcal{L}_{\mathbb{R}^{d}}$, and it yields an isometry between $\mathcal{L}_{\mathbb{R}^{d}}$ and all of $L_{2}\left(\mathbb{R}^{d}\right)$.

Proof. It is evident that $C$ is isometric, and thus $C$ extends to $\mathcal{L}_{\mathbb{R}^{d}}$. But the density of $C\left(\mathcal{L}_{\Omega}\right)$ in $L_{2}\left(\mathbb{R}^{d}\right)$ does not follow from abstract Hilbert space arguments. We thus need an additional analytic argument. We first prove the assertion for continuous $\hat{\Phi}$ with $\hat{\Phi}>0$ on $\mathbb{R}^{d} \backslash\{0\}$. Let some function $f \in L_{2}\left(\mathbb{R}^{d}\right)$ and some $\varepsilon>0$ be given. Then there is a compactly supported $C^{\infty}$ function $g \in L_{2}\left(\mathbb{R}^{d}\right)$ such that $\|f-g\|_{2} \leq \varepsilon$. This is a standard argument in Fourier analysis, obtainable by convolution and "chopping near infinity". Now define $\hat{u}:=g / \sqrt{\hat{\Phi}}$ on $\mathbb{R}^{d}$, where the (possible) singularity of $\hat{\Phi}$ at zero does no harm. Clearly $\hat{u}$ is continuous and 
compactly supported, thus in $L_{2}\left(\mathbb{R}^{d}\right)$ and $u$ is band-limited, of exponential type, and in $L_{2}\left(\mathbb{R}^{d}\right)$. We now invoke the multivariate sampling theorem to recover $u$ exactly from its function values on a grid in $\mathbb{R}^{d}$ with spacing $h$, where $h$ is sufficiently small and related to the support of $\hat{u}$.

Thus we have

$$
u(x)=\sum_{j \in \mathbb{Z}^{d}} u(j h) \operatorname{Sinc}_{d}\left(\frac{x-j h}{h}\right), \quad x \in \mathbb{R}^{d}
$$

where

$$
\operatorname{Sinc}_{d}\left(x_{1}, \ldots, x_{d}\right)=\prod_{j=1}^{d} \frac{\sin \pi x_{j}}{\pi x_{j}}
$$

and

$$
\hat{u}(\omega)=\sum_{j \in \mathbb{Z}_{d}} u(j h) e^{i h j^{T} \omega}, \quad \omega \in \mathbb{R}^{d},
$$

has the form $\hat{u}=\widehat{\lambda_{u}}$ for the functional

$$
\lambda_{u}(v)=\sum_{j \in \mathbb{Z}^{d}} v(j h) u(j h) .
$$

We now have to make sure that $\lambda_{u} \in \mathcal{L}_{\mathbb{R}^{d}}$. If this is done, we are finished, because $C\left(\lambda_{u}\right)=g$ and

$$
\|f-\sqrt{\hat{\Phi}} \widehat{\lambda u}\|_{2}=\|f-g\|_{2} \leq \varepsilon .
$$

For all $p \in \mathbb{P}_{m}^{d}$ we have to show that $\lambda_{u}(p)=0$. By a standard argument in Fourier analysis this requires a zero of order at least $m$ of $\hat{u}$ at zero. But our assumption (4.6) on $\hat{\Phi}$ and the minimality of $m$ in (4.5) imply that $\hat{u}$ has a zero of order at least

$$
\frac{1}{2}\left(d+\beta_{0}\right)>\frac{1}{2}(d+2 m-2)=m-1+\frac{d}{2},
$$

thus of order $\geq m$.

We finally have to check that

$$
\left\|\lambda_{u}\right\|_{\Phi}^{2}=\|\sqrt{\hat{\Phi}} \widehat{\lambda u}\|_{2}^{2}=\|\sqrt{\hat{\Phi}} \hat{u}\|_{2}^{2}=\|g\|_{2}^{2}<\infty,
$$

and this concludes the proof for $\hat{\Phi}>0$.

Now let $\hat{\Phi}$ be positive up to a set of Lebesgue measure zero. We cover the set of zeros by intervals $I_{k}$, where $k$ varies over some index set $K$ and the total area $\sum_{k}\left|I_{k}\right|$ is less than some given $\delta$. Now let $\hat{\Phi}_{\delta}(\omega) \geq \hat{\Phi}(\omega)$ be a strictly positive continuous function that differs from $\hat{\Phi}$ only on the $I_{k}$. Then $\hat{\Phi}_{\delta}$ will also satisfy our assumptions, and we can use (4.1) in the form

$$
(\mu, \lambda)_{\Phi_{\delta}}:=(2 \pi)^{-d / 2} \int_{\mathbb{R}^{d}} \hat{\Phi}_{\delta}(\omega) \sigma_{\lambda}(\omega) \overline{\sigma_{\mu}(\omega)} d \omega
$$

as a definition of an inner product, but we do not need $\Phi_{\delta}$ explicitly.

Now we approximate a given $f \in L_{2}\left(\mathbb{R}^{d}\right)$ by some $\sqrt{\hat{\Phi}_{\delta}} \hat{\lambda}$ up to $\varepsilon / 2$ in the $L_{2}$ norm, picking a suitable $\lambda$ for each $\delta$ and $\varepsilon$. Then

$$
\|f-\sqrt{\hat{\Phi}} \hat{\lambda}\|_{2} \leq\left\|f-\hat{\lambda} \sqrt{\hat{\Phi}_{\delta}}\right\|_{2}+\left\|\hat{\lambda}\left(\sqrt{\hat{\Phi}_{\delta}}-\sqrt{\hat{\Phi}}\right)\right\|_{2}
$$


and

$$
\begin{aligned}
\left\|\hat{\lambda}\left(\sqrt{\hat{\Phi}_{\delta}}-\sqrt{\hat{\Phi}}\right)\right\|_{2}^{2} & =\left\|\hat{\lambda} \sqrt{\hat{\Phi}_{\delta}}\left(1-\sqrt{\hat{\Phi} / \hat{\Phi}_{\delta}}\right)\right\|_{2}^{2} \\
& \leq \sum_{k} \int_{I_{k}}|\hat{\lambda}(\omega)|^{2} \hat{\Phi}_{\delta}(\omega) d \omega .
\end{aligned}
$$

The full integral

$$
\int_{\mathbb{R}^{d}}|\hat{\lambda}(\omega)|^{2} \hat{\Phi}_{\delta}(\omega) d \omega=\left\|\hat{\lambda} \sqrt{\hat{\Phi}_{\delta}}\right\|_{2}^{2}
$$

can be bounded independent of $\delta$, because it approximates $\|f\|_{2}^{2}$. Thus we are able to pick $\delta$ small enough to guarantee

$$
\sum_{k} \int_{I_{k}}|\hat{\lambda}(\omega)|^{2} \hat{\Phi}_{\delta}(\omega) d \omega \leq \varepsilon / 2
$$

yielding an overall bound $\|f-\sqrt{\hat{\Phi}} \hat{\lambda}\|_{2} \leq \varepsilon$.

We now use Theorem 4.1 to characterize the native space $\mathcal{F}_{\mathbb{R}^{d}}$ for $\Phi$ via $L_{2}\left(\mathbb{R}^{d}\right)$. Starting with an arbitrary $h \in L_{2}\left(\mathbb{R}^{d}\right)$, the function

$$
f_{h}(x):=\left(h, C \delta_{(x)}\right)_{L_{2}\left(\mathbb{R}^{d}\right)}
$$

is in $\mathcal{G}_{\mathbb{R}^{d}}$, because the equality

$$
\lambda f_{h}=(\hat{h}, C \lambda)_{L_{2}\left(\mathbb{R}^{d}\right)}
$$

follows easily from (4.7) for all $\lambda \in\left(\mathbb{P}_{m}^{d}\right)^{\perp}$. We can rewrite (4.7) as

$$
\begin{aligned}
f_{h}(x) & =(2 \pi)^{-d} \int_{\mathbb{R}^{d}} \hat{h}(\omega) \sqrt{\hat{\Phi}(\omega)}\left(e^{i x^{T} \omega}-\sum_{j=1}^{Q} p_{j}(x) e^{i \xi_{j}^{T} \omega}\right) d \omega \\
& =(2 \pi)^{-d} \int_{\mathbb{R}^{d}} \hat{f}_{h}(\omega)\left(e^{i x^{T} \omega}-\sum_{j=1}^{Q} p_{j}(x) e^{i \xi_{j}^{T} \omega}\right) d \omega
\end{aligned}
$$

where we define

$$
\hat{f}_{h}:=h \sqrt{\hat{\Phi}}
$$

which is fully consistent with the usual notation for Fourier transforms when $m=0$.

Theorem 4.2. The native space $\mathcal{F}_{\mathbb{R}^{d}}$ for a conditionally positive definite function of order $m$ on $\mathbb{R}^{d}$ coincides with the space of all functions $f$ on $\mathbb{R}^{d}$ that can be written as

$$
f(x)=(2 \pi)^{-d} \int_{\mathbb{R}^{d}} \hat{f}(\omega)\left(e^{i x^{T} \omega}-\sum_{j=1}^{Q} p_{j}(x) e^{i \xi_{j}^{T} \omega}\right) d \omega
$$

plus polynomials from $\mathbb{P}_{m}^{d}$, and where $\hat{f}$ is a function that satisfies

$$
\hat{f} / \sqrt{\hat{\Phi}} \in L_{2}\left(\mathbb{R}^{d}\right) .
$$

The inner product on $\mathcal{F}_{\mathbb{R}^{d}}$ can be rewritten as

$$
(f, g)_{\Phi}=(2 \pi)^{-d} \int_{\mathbb{R}^{d}} \frac{\hat{f}(\omega) \overline{\hat{g}(\omega)}}{\hat{\Phi}(\omega)} d \omega .
$$


Note that (4.8) yields $f\left(\xi_{j}\right)=0$, thus picking up the special assignment of function values that we already used in (3.3). Given $f \in \mathcal{F}_{\mathbb{R}^{d}}$, the function $\hat{f}$ is uniquely defined by

$$
\hat{f}=\sqrt{\hat{\Phi}}\left(C \circ B^{-1}\right) f .
$$

There also is a way to describe the action of functionals $\lambda \in \mathcal{L}_{\mathbb{R}^{d}}$ on functions $f \in \mathcal{F}_{\mathbb{R}^{d}}$ via

$$
\lambda(f)=(2 \pi)^{-d} \int_{\mathbb{R}^{d}}(C \lambda)(\omega) \frac{\hat{f}(\omega)}{\sqrt{\hat{\Phi}(\omega)}} d \omega=\left(C \lambda, C B^{-1} f\right)_{L_{2}\left(\mathbb{R}^{d}\right)}
$$

where $C \lambda=\sqrt{\hat{\Phi}} \hat{\lambda}$ is a function in $L_{2}\left(\mathbb{R}^{d}\right)$.

We now introduce a $\mathbb{P}_{m}^{d}$-nondegenerate subset $\Omega$ with $\Xi \subseteq \Omega \subseteq \mathbb{R}^{d}$, and we want to study $\mathcal{F}_{\Omega}$ and $\mathcal{L}_{\Omega}$ instead of $\mathcal{F}_{\mathbb{R}^{d}}$ and $\mathcal{L}_{\mathbb{R}^{d}}$. This does not yield handy results directly, because restriction of supports does not nicely affect the Fourier transforms.

In particular, the condition $\operatorname{supp}(\lambda) \subseteq \Omega$ does not enter directly into $C(\lambda)=$ $\sqrt{\hat{\Phi}} \hat{\lambda} \in L_{2}\left(\mathbb{R}^{d}\right)$, but it trivially affects $\lambda=(\hat{\lambda})^{\vee}=(C(\lambda) / \sqrt{\hat{\Phi}})^{\vee}$, where $\vee$ stands for the inverse Fourier transform. Formally, we have

$$
\frac{C(\lambda)}{\sqrt{\hat{\Phi}}}=\hat{\lambda}=\frac{\hat{f}_{\lambda}}{\hat{\Phi}}
$$

and these functions are in $L_{\infty}\left(\mathbb{R}^{d}\right)$ for $\lambda \in\left(\mathbb{P}_{m}^{d}\right)^{\perp}$, but are not nicely controllable for general $\lambda \in \mathcal{L}_{\Omega}$. Since we want to take inverse Fourier transforms, we have to add an assumption that restricts the admissible functions, and this will lead to the subspace

$$
\mathcal{H}_{\Omega}:=\left\{f \in \mathcal{F}_{\Omega}: \hat{f}_{e} / \hat{\Phi} \in L_{2}\left(\mathbb{R}^{d}\right)\right\}
$$

of $\mathcal{F}_{\Omega}$.

In most cases the space $\mathcal{H}_{\Omega}$ is related to the native space of a conditionally positive definite function $\Psi$ that equals $\Phi * \Phi$ or generalizes it. In cases where $\Phi * \Phi$ is a meaningful convolution in $\mathbb{R}^{d}$, this relation is obvious except for the boundary conditions. Note that $\mathcal{H}_{\Omega}$ inherits the boundary conditions from $\mathcal{F}_{\Omega}$ (i.e. those defined by $\Phi)$, while the native space for $\Phi * \Phi$ will have somewhat different boundary conditions.

Since we want to keep cubic splines as our major example, we still have to treat cases where $\Phi * \Phi$ does not directly make sense. In fact, if $\hat{\Phi}$ satisfies bounds like

$$
\begin{aligned}
|\hat{\Phi}(\omega)| & \leq C_{0}\|\omega\|_{2}^{-d-\beta_{0}}, \quad \omega \text { around zero, } \\
|\hat{\Phi}(\omega)| & \leq C_{\infty}\|\omega\|_{2}^{-d-\beta_{\infty}}, \quad \omega \text { around infinity, } \\
\beta_{0} & \leq 2 m \\
\beta_{\infty} & >0
\end{aligned}
$$

then one can directly see that $\sqrt{\hat{\Phi}} \hat{\lambda} \in L_{2}\left(\mathbb{R}^{d}\right)$ for all $\lambda \in\left(\mathbb{P}_{m}^{d}\right)^{\perp}$, making the above assumptions valid for practically every case in use. If we assume 
somewhat more, i.e.,

$$
\beta_{\infty}>d / 2, \quad 2 \beta_{0}+d \leq 2 n
$$

then $\hat{\Phi} \hat{\mu} \in L_{2}\left(\mathbb{R}^{d}\right)$ for all $\mu \in\left(\mathbb{P}_{n}^{d}\right)^{\perp}$, and one can define the quadratic form

$$
(2 \pi)^{-d} \int_{\mathbb{R}^{d}}(\hat{\Phi}(\omega))^{2} \hat{\lambda}(\omega) \overline{\hat{\mu}(\omega)} d \omega
$$

for all $\lambda, \mu \in\left(\mathbb{P}_{n}^{d}\right)^{\perp}$. One way to use (4.9) to define a (generalized) conditional positive definite function of order $n$ is to introduce functionals of the form $\delta_{(x)}$ like in (3.2), but using a $\left(\mathbb{P}_{n}^{d}\right)$-unisolvent set. Then

$$
\Psi(x, y):=(2 \pi)^{-d} \int_{\mathbb{R}^{d}}(\hat{\Phi}(\omega))^{2} \hat{\delta}_{(x)}(\omega) \overline{\hat{\delta}}_{(y)}(\omega) d \omega
$$

is a generalized conditionally positive definite function of order $n$ on $\mathbb{R}^{d}$ in the sense that (4.9) takes the form

$$
\sum_{j=1}^{M} \sum_{k=1}^{N} \lambda_{j} \mu_{k} \Psi\left(x_{j}, y_{k}\right)
$$

and defines a positive definite quadratic form on $\left(\mathbb{P}_{n}^{d}\right)^{\perp}$.

In the model case $\Phi_{\beta}(\omega)=\|\omega\|_{2}^{\beta}$ on $\mathbb{R}^{d}$ one has $\beta=\beta_{0}=\beta_{\infty}$ and $\hat{\Phi}_{\beta}(\omega)=$ $c\|\omega\|_{2}^{-d-\beta}$. Then

$$
\hat{\Phi}_{\beta}^{2}(\omega)=c^{2}\|\omega\|_{2}^{-2 d-2 \beta}=\hat{\Phi}_{2 \beta+d}(\omega)
$$

up to a multiplicative constant. While $\Phi_{\beta}$ is conditionally positive definite of order $m \geq \beta / 2$ on $\mathbb{R}^{d}$ the function $\Phi_{2 \beta+d}$ has order $n \geq \beta+d / 2$. If we take $d=1$ and $\beta=3$, we have $m=2,2 \beta+d=7, n=4$. The boundary conditions for cubics require functions to be linear outside $\Omega=[a, b]$, while the boundary conditions for septics require cubics outside $\Omega$. The smoothness of functions $f$ in the related native spaces is $f^{\prime \prime} \in L_{2}$ for cubics and $f^{(4)} \in L_{2}$ for septics. But the appropriate space for proving approximation order $h^{7 / 2}=h^{(2 \beta+d) / 2}$ of interpolants requires $f^{(4)} \in L_{2}$, i.e. smoothness for septics, and $f^{(3)}(x)=f^{(2)}(x)=0$ for $x$ outside $\Omega$, i.e. boundary conditions for cubics. This is why we defined $\mathcal{H}_{\Omega}$ as a subspace of $\mathcal{F}_{\Omega}$ : it then inherits the boundary conditions from $\Phi$, but the smoothness is related to $\Phi * \Phi$. In this example, it contains functions $f$ with $f^{(4)} \in L_{2}$ which are linear outside of $[a, b]$.

Given $f \in \mathcal{H}_{\Omega}$, we have the two functions

$$
h_{f}=\frac{\hat{f}_{e}}{\hat{\Phi}}, \quad g_{f}=\frac{\hat{f}_{e}}{\sqrt{\hat{\Phi}}}
$$

in $L_{2}\left(\mathbb{R}^{d}\right)$. Now for any $v \in L_{2}\left(\mathbb{R}^{d}\right) \cap \mathcal{F}_{\mathbb{R}^{d}}$ we get

$$
\begin{aligned}
\int_{\mathbb{R}^{d}} h_{f} v & =(2 \pi)^{-d} \int h_{f} \hat{v}=(2 \pi)^{-d} \int \frac{\hat{f}_{e}}{\hat{\hat{\Phi}}} \hat{v} \\
& =(2 \pi)^{-d} \int \frac{\hat{f}_{e}}{\sqrt{\hat{\Phi}}} \frac{\hat{v}}{\sqrt{\hat{\Phi}}}=\left(f_{e}, v\right)_{\Phi}=(f, v)_{\Phi}
\end{aligned}
$$

such that the functional

$$
v \mapsto(f, v)_{\Phi}=\left(h_{f}, v\right)_{L_{2}\left(\mathbb{R}^{d}\right)}
$$


is continuous in $L_{2}\left(\mathbb{R}^{d}\right) \cap \mathcal{F}_{\Omega}$ and represented by $h_{f}$. Taking $v \in L_{2}\left(\mathbb{R}^{d}\right) \cap \mathcal{F} \perp$ we get

$$
\int_{\mathbb{R}^{d}} h_{f} v=0
$$

and from this variational equation we want to conclude that $h_{f}$ is supported in $\Omega$. Note that for $f=f_{\lambda} \in \mathcal{F}_{\Omega}$ with $\lambda \in\left(\mathbb{P}_{m}^{d}\right)_{\Omega}^{\perp}$ we formally have

$$
\hat{f}_{\lambda}=\hat{\lambda} \hat{\Phi}, \quad h_{f}=\hat{\lambda}
$$

such that $h_{f}$ coincides up to a factor with the functional $\lambda$ that is indeed supported in $\Omega$. This makes perfect sense in the context of distributions, but it requires some additional analysis to carry this over to the case of $f \in \mathcal{H}_{\Omega}$.

Theorem 4.3. If $\hat{\Phi}$ decays not faster than algebraically at infinity (i.e. if $\Phi$ is of limited smoothness), then for any $f \in \mathcal{H}_{\Omega}$ and any closed set $\Omega \subseteq \mathbb{R}^{d}$ the $L_{2}$ function $(\hat{f} / \hat{\Phi})^{\vee}=h_{f}$ vanishes almost everywhere outside $\Omega$.

Proof. Our assumptions imply that all $C_{0}^{\infty}$ functions $v$ with support outside $\Omega$ are in $\mathcal{F}_{\Omega}^{\perp} \cap L_{2}\left(\mathbb{R}^{d}\right)$. Thus

$$
\int_{\mathbb{R}^{d}} h_{f} v=0
$$

for all such functions, proving that $h_{f}$ vanishes almost everywhere outside $\Omega$.

The converse is also true.

Theorem 4.4. If $f \in \mathcal{F}_{\mathbb{R}^{d}}$ has the property that $(\hat{f} / \hat{\Phi})^{\vee}=h_{f}$ is in $L_{2}\left(\mathbb{R}^{d}\right)$ with support in $\Omega$, then $f \in \mathcal{H}_{\Omega}$.

Proof. We need to show that $f$ is orthogonal to $\mathcal{F}_{\Omega}^{\perp}$. Taking any $g \in \mathcal{F}_{\Omega}^{\perp} \cap L_{2}\left(\mathbb{R}^{d}\right)$ we can assign function values to $g$ modulo $\mathbb{P}_{m}^{d}$ such that $\left.g\right|_{\Omega}=\{0\}$. Then

$$
(f, g)_{\Phi}=\int_{\mathbb{R}^{d}} \frac{\hat{f} \overline{\hat{g}}}{\hat{\Phi}}=\int_{\mathbb{R}^{d}} \hat{h}_{f} \hat{\hat{g}}=\int_{\Omega} h_{f} g=0 .
$$

The rest follows from density of $L_{2}\left(\mathbb{R}^{d}\right) \cap \mathcal{F}_{\mathbb{R}^{d}}$ in $\mathcal{F}_{\mathbb{R}^{d}}$ by chopping Fourier transforms.

The additional assumption on $\hat{\Phi}$ excludes cases with exponential decay of the Fourier transform (e.g. multiquadrics and Gaussians). To include these one needs a sufficiently large space of test functions with even faster decay of Fourier transforms. But since in these cases the error bounds for interpolation are of exponential type, our goal of "squaring" the error bounds would not yield a significant improvement anyway. We thus skip over further elaboration of details for such cases.

We conclude this section by a sketch of the functional-analytic background of the space $\mathcal{H}_{\Omega}$. The latter can be redefined as the subspace of functions $f \in \mathcal{F}_{\Omega}$ that make the functional $v \mapsto(f, v)_{\Phi}$ continuous on $L_{2}\left(\mathbb{R}^{d}\right)$, such that there is a function $h_{f} \in L_{2}\left(\mathbb{R}^{d}\right)$ with $h_{f}=0$ outside $\Omega$ and

$$
(f, v)_{\Phi}=\left(h_{f}, v\right)_{L_{2}\left(\mathbb{R}^{d}\right)}=\left(\hat{h}_{f}, \hat{v}\right)_{L_{2}(\Omega)}=(L f, L v)_{L_{2}\left(\mathbb{R}^{d}\right)}
$$

for all $v \in L_{2}\left(\mathbb{R}^{d}\right) \cap \mathcal{F}_{\Omega}$, where

$$
L v=\frac{\hat{v}}{\sqrt{\hat{\Phi}}} \in L_{2}\left(\mathbb{R}^{d}\right) .
$$


Thus $h_{f}=L^{*} L f$ with the $L_{2}$-adjoint $L^{*}$ of $L$, so that (4.11) can be rewritten as

$$
(f, v)_{\Phi}=\left(L^{*} L f, v\right)_{L_{2}\left(\mathbb{R}^{d}\right)}=(L f, L v)_{L_{2}\left(\mathbb{R}^{d}\right)} .
$$

Here the pseudodifferential operator $L$ is associated to $\sqrt{\hat{\Phi}}$, while $L^{*} L$ is associated to $\hat{\Phi}$. This is another analogy with the univariate spline case, where $L$ usually is a differential operator of order $m$ and the native space consists of $f$ with $L f \in$ $L_{2}$, while improved convergence holds for $f$ with $L^{*} L f \in L_{2}$ plus homogeneous boundary conditions concerning the derivatives of order $m$ to $2 m-1$.

Theorem 4.5. Under the hypotheses of Theorem 4.3 the a priori estimate

$$
\|f\|_{\Phi}^{2} \leq\left\|h_{f}\right\|_{L_{2}(\Omega)}\|f\|_{L_{2}(\Omega)}
$$

holds for all $f \in \mathcal{H}_{\Omega} \cap L_{2}(\Omega)$.

Proof. From (4.10) and Theorem 4.3 we get

$$
\|f\|_{\Phi}^{2}=\left(h_{f}, f\right)_{L_{2}\left(\mathbb{R}^{d}\right)}=\left(h_{f}, f\right)_{L_{2}(\Omega)}
$$

for all $f \in \mathcal{H}_{\Omega} \cap L_{2}\left(\mathbb{R}^{d}\right) \cap \mathcal{F}_{\mathbb{R}^{d}}$. This extends by continuity to all $f \in \mathcal{H}_{\Omega} \cap L_{2}(\Omega)$.

Corollary 4.6. Under the assumptions of Theorem 4.3 and with the notation

$$
\|f\|_{\Phi * \Phi}:=\left\|\frac{\hat{f}_{e}}{\hat{\Phi}}\right\|_{L_{2}\left(\mathbb{R}^{d}\right)}=\left\|\left(\hat{f}_{e} / \hat{\Phi}\right)^{\vee}\right\|_{L_{2}(\Omega)}
$$

for $f \in \mathcal{H}_{\Omega}$ we have

$$
\left|(f, v)_{\Phi}\right| \leq\|f\|_{\Phi * \Phi}\|v\|_{L_{2}(\Omega)}
$$

for all $v \in L_{2}(\Omega) \cap \mathcal{F}_{\mathbb{R}^{d}}$ and $f \in \mathcal{H}_{\Omega}$.

Proof. Same as for Theorem 4.5.

\section{IMPROVED ERROR BOUNDS}

We now want to assemble the results of Sections 3 and 4 into a proof of the error bound (2.12).

Theorem 5.1. Let $\Phi: \mathbb{R}^{d} \rightarrow \mathbb{R}$ be a continuous conditionally positive definite function of order $m \geq 0$. It should have a nonnegative Fourier transform $\hat{\Phi}$ on $\mathbb{R}^{d} \backslash\{0\}$ in the sense of Section 4, and the decay of $\hat{\Phi}(\omega)$ at infinity should not be faster than some $\|\omega\|^{-\alpha}$ for $\alpha>0$. Such a function $\Phi$ leads to a native space $\mathcal{F}=\mathcal{F}_{\mathbb{R}^{d}}$ of functions $f$ on $\mathbb{R}^{d}$ which have Fourier transforms $\hat{f}$ in the sense of (4.8) such that $\hat{f} / \sqrt{\hat{\Phi}} \in L_{2}\left(\mathbb{R}^{d}\right)$, the norm being

$$
\|f\|_{\Phi}^{2}=(2 \pi)^{-d} \int_{\mathbb{R}^{d}} \frac{|\hat{f}(\omega)|^{2}}{\hat{\Phi}(\omega)} d \omega
$$

For functions $f \in \mathcal{F}$ there is the usual error bound (2.11) with $P_{X}$ defined by (2.10). Then for any compact set $\Omega \subset \mathbb{R}^{d}$ there is a subspace $\mathcal{H}_{\Omega}$ of $\mathcal{F}_{\mathbb{R}^{d}}$ with norm

$$
\|f\|_{\Phi * \Phi}^{2}=(2 \pi)^{-d} \int \frac{|\hat{f}(\omega)|^{2}}{\hat{\Phi}^{2}(\omega)} d \omega
$$

such that the error bound

$$
\left|\left(f-s_{X, f}\right)(x)\right| \leq\|f\|_{\Phi * \Phi} P_{X}(x)\left\|P_{X}\right\|_{L_{2}(\Omega)}
$$


holds for all $f \in \mathcal{H}_{\Omega}$ and $x \in \Omega$, where $s_{X, f}$ interpolates $f$ on a discrete $\mathbb{P}_{m}^{d}-$ nondegenerate $X$ of $\Omega$. The subspace $\mathcal{H}_{\Omega}$ consists of functions $f \in \mathcal{F}_{\mathbb{R}^{d}}$ such that $(5.1)$ is finite and $(\hat{f} / \hat{\Phi})^{\vee}$ is a function in $L_{2}$ whose $L_{2}$-Fourier transform is supported in $\Omega$.

Proof. We first use the standard pointwise error bound (2.11) in its special form

$$
\left|\left(f-s_{X, f}\right)(x)\right| \leq\left\|f-s_{X, f}\right\|_{\Phi} P_{X}(x)
$$

to prove $f-s_{X, f} \in L_{2}(\Omega)$ via

$$
\left\|f-s_{X, f}\right\|_{L_{2}(\Omega)}^{2} \leq\left\|f-s_{X, f}\right\|_{\Phi}^{2}\left\|P_{X}\right\|_{L_{2}(\Omega)}^{2}
$$

for all $f \in \mathcal{F}_{\mathbb{R}^{d}}$. Note that $P_{X}$ is continuous due to (2.10). Then we use the minimum-norm property of the interpolant $s_{X, f}$ in the form

$$
\left(f-s_{X, f}, s_{X, f}\right)_{\Phi}=0
$$

which also follows from Corollary 3.4, since $f-s_{X, f}$ takes the value zero on the points where the functional associated to $s_{X, f}$ is supported. This yields

$$
\begin{aligned}
\left\|f-s_{X, f}\right\|_{\Phi}^{2} & =\left(f-s_{X, f}, f-s_{X, f}\right)_{\Phi}=\left(f, f-s_{X, f}\right)_{\Phi} \\
& \leq\|f\|_{\Phi * \Phi}\left\|f-s_{X, f}\right\|_{L_{2}(\Omega)} \leq\|f\|_{\Phi * \Phi}\left\|f-s_{X, f}\right\|_{\Phi}\left\|P_{X}\right\|_{L_{2}(\Omega)}
\end{aligned}
$$

due to (4.12) and (5.4). Cancelling a factor $\left\|f-s_{X, f}\right\|_{\Phi}$, we get

$$
\left\|f-s_{X, f}\right\|_{\Phi} \leq\|f\|_{\Phi * \Phi}\left\|P_{X}\right\|_{L_{2}(\Omega)},
$$

and the assertion follows from (5.3).

\section{REFERENCES}

[1] Ahlberg, J.H., Nilson, E.N. and Walsh, J.L. The theory of splines and their applications, Mathematics in Science and Engineering (38), Academic Press, 1967. MR 39:684

[2] Iske, A. Characterization of function spaces associated with conditionally positive definite functions, Mathematical Methods for Curves and Surfaces, (M. Daehlen, T. Lyche and L.L. Schumaker, eds.), Vanderbilt University Press, Nashville, TN, pp. 265-270, 1995. MR 96f: 65003

[3] Iske, A. Reconstruction of functions from generalized Hermite-Birhoff data, Approximation Theory VIII, (C.K. Chui and L.L. Schumaker, eds.), World Scientific, Singapore, pp. 257-264, 1995. CMP 98:01

[4] Madych, W.R. and Nelson, S.A. Error bounds for multiquadric interpolation, Approximation Theory VI (C.K. Chui and L.L. Schumaker and J.D. Ward, eds.), Academic Press, Boston, pp. 413-416, 1989. MR 91j:41002

[5] Madych, W.R. and Nelson, S.A. Multivariate interpolation and conditionally positive definite functions. II, Math. Comp. 54 (1990), pp. 211-230. MR 90e:91007

[6] Madych, W.R. and Nelson, S.A. Bounds on multivariate polynomials and exponential error estimates for multiquadric interpolation, Journal of Approximation Theory 70 (1992), pp. 94-114. MR 93f:41009

[7] Schaback, R. Error estimates and condition numbers for radial basis function interpolation, Advances in Computational Mathematics 3 (1995), pp. 251-264. MR 96a:41004

[8] Schaback, R. and Wu, Z. Operators on radial functions, J. of Comp. and Appl. Math. 73 (1996), pp. 257-270. MR 97g:42002

[9] Schumaker, L.L. Spline Functions: Basic Theory, Wiley-Interscience, 1981. MR 82j:41001

[10] $\mathrm{Wu}, \mathrm{Z}$. and Schaback, R., Local error estimates for radial basis function interpolation of scattered data, IMA Journal of Numerical Analysis 13 (1993), pp. 13-27. MR 93m:65012

Institut für Numerische und Angewandte Mathematik, Georg-August-Universität, Lotzestrasse 16-18, 37083, Göttingen, Germany 